\title{
On the Mass Neutrino Phase along the geodesic line and the null line in curved and flat spacetime
}

\author{
C.M. Zhang ${ }^{1,2}$, and A.Beesham ${ }^{3}$ \\ 1. Research Centre for Theoretical Astrophysics, School of Physics, \\ University of Sydney, NSW 2006, Australia \\ zhangcm@physics.usyd.edu.au \\ 2. High Energy Section, ICTP, strada Costiera 11, 34100 Trieste, Italy \\ 3. Department of Mathematical Sciences, University of Zululand, South Africa
}

\begin{abstract}
On the mass neutrino phase calculations along both the particle geodesic line and the photon null line, there exists a double counting, factor of 2 , when comparing the geodesic phase with the null phase. Moreover, we compare the phase calculations among the same energy description, the same momentum description by means of the Minkowski diagram, and obtain the practical equivalence of these two descriptions. On the same velocity description, although it does not correspond to a reality of physical process, we still indicate its phase calculation in the Minkowski diagram, which has the same result as those of same energy and same momentum cases. Further, in the curved spacetime, we also prove the existence of the double counting of the geodesic phase to the null phase. Our conclusions are same as others' results by the different methods.
\end{abstract}




\section{Introduction}

There have been many topics issued on the quantum mechanics of the neutrino oscillation in the flat spacetime $[1,2,3,4,5]$ and in the curved spacetime $[6,7,8,9,10,11,12,13]$, most of them centered on the understanding of the quantum coherence condition between the various mass-eigenstates, as well as on the calculations of the phase factor in the spacetime. The mass neutrino oscillation problem is connected basically to the phase difference between different mass-eigenstate, a property intimately related to the basic principles of quantum mechanics. In the standard treatment of the neutrino oscillation, the condition of same-energy (samemomentum) with different momenta (energies) of the mass-eigenstates is introduced [2, 4, $5,14,15,16]$, which arises the same practically applied massive neutrino oscillation phase, however it is indicated that the unambiguous theoretical description of the massive neutrino oscillation phase will be involved in the wave packet formalism and not in the plane wave approximation [17]. Further, it is shown that the same energy or same momentum description is just the arbitrariness of the choice of the Lorentz frame in which it is valid, nothing to do with the physical argument [18]. The change of flavors is described by the oscillation length, and it is usually claimed that both conditions (same-energy and same-momentum) present practically the same neutrino oscillation results. Sometimes, a source-dependent condition is added [19, 20, 21], which implies in giving up either the same-energy or the same-momentum prescription. This calculation of the neutrino oscillation phase, however, yields the same result of the standard treatment. Furthermore, the velocity difference of the various masseigenstates results in a spacetime separation for neutrinos of different flavor [9, 22, 23], which is another source of confusion on the interference condition for the different neutrino. The same velocity description has been paid much attention [24, 25, 26], but this prescription is pointed out to be forbidden kinematically [27]. Actually, considerable confusion has arisen in the description, interpretation and understanding of the neutrino oscillation, a problem which involves the fundamental principles of both quantum mechanics and special relativity, such as the uncertainty principle, the superposition principle, and simultaneity problems because the mass neutrinos are high energy quantum objects. Moreover it is often noted the factor of 2 of the neutrino phase calculations in the flat spacetime [22, 28] and in the curved spacetime $[9,10]$, which is believed to be the consideration of the space phase or the time phase [9], as well as the arrival time difference of the two mass neutrinos [10].

In the Minkowski diagram of flat spacetime, we discuss how the same-energy, the samemomentum of the neutrino phase present the same practical result, and the relation between the arrival time difference and the double counting of the phase, and further, we extend our discussions to the curved spacetime. We also calculate the phase factor in the case of the same velocity description although this process is not physically realized. Here we stress that all our calculations are based on the plane wave treatment of the neutrino, otherwise 
the wave packet should be considered [29]. We set $\hbar=c=1$ throughout this article.

\section{The Standard Treatment of the Neutrino Oscillation}

In the standard treatment of the neutrino oscillation, if two generations are taken into account, the neutrino flavor-state is written as

$$
\left|\nu_{\alpha}(x, t)\right\rangle=\sum_{j} U_{\alpha j} \exp \left[i \Phi_{j}\right]\left|\nu_{j}\right\rangle
$$

where

$$
(U)=U_{\alpha j}=\left(\begin{array}{cc}
\cos \theta & \sin \theta \\
-\sin \theta & \cos \theta
\end{array}\right),
$$

with $\theta$ the mixing angle. In vector form,

$$
\left(\begin{array}{l}
\left|\nu_{e}(x, t)\right\rangle \\
\left|\nu_{\mu}(x, t)\right\rangle
\end{array}\right)=\exp (i \boldsymbol{\Phi}) \mathbf{U}\left(\begin{array}{l}
\left|\nu_{1}\right\rangle \\
\left|\nu_{2}\right\rangle
\end{array}\right),
$$

where

$$
(\exp (i \boldsymbol{\Phi}))=\left(\begin{array}{cc}
\exp \left(i \Phi_{1}\right) & 0 \\
0 & \exp \left(i \Phi_{2}\right)
\end{array}\right)
$$

with $\Phi_{j}$ the eigenvalue of the phase operator [6]

$$
\Phi_{j}=\int\left(E_{j} d t-P_{j} d x\right)
$$

Here, flavor (mass) indices are expressed by Greek (Latin) letters, and $\nu_{e}$ and $\nu_{\mu}$ are represented respectively by $\nu_{1}$ and $\nu_{2}$. The matrix elements $U_{\alpha j}$ comprise the transformation between the flavor and mass basis. Now, we suppose a pure flavor-state electron neutrino $\left|\nu_{e}\right\rangle$ is at the initial source position $\mathbf{A}(x=0$ and $t=0)$. The mass eigenstates are taken to be the eigenstates of energy (momentum). The momentum (energy) then satisfies the mass shell relation

$$
\mathbf{P}=\sqrt{\mathbf{E}^{2}-\mathbf{m}^{2}} \approx \mathbf{E}-\frac{\mathbf{m}^{2}}{2 \mathbf{E}},
$$

where $\mathbf{m}$ is the rest-mass operator corresponding to the mass eigenvalue $m_{j}$ of the eigenstate $\left|\nu_{j}\right\rangle[6]$.

The oscillation probability $\mathcal{P}$ from flavor $\left|\nu_{e}\right\rangle$ at the source position $\mathbf{A}$, to flavor $\left|\nu_{\mu}\right\rangle$ at the detector position $\mathbf{B}(x=L)$ is given by [5],

$$
\mathcal{P}\left(\nu_{e} \rightarrow \nu_{\mu}\right)=\left|\left\langle\nu_{\mu} \mid \nu_{e}(x, t)\right\rangle\right|^{2}=\sin ^{2} 2 \theta \cos \frac{\Delta \Phi}{2},
$$

where

$$
\Delta \Phi=\Phi_{2}-\Phi_{1}
$$


is the phase difference between the different mass states. The oscillation length is defined by taking $\Delta \Phi=2 \pi$. From the standard treatment of the neutrino oscillation, the common momentum (energy), and the approximation condition (6) lead to a phase difference $\Delta \Phi$ and an oscillation length $L_{\text {osc }}^{S}$ given respectively by [5]

$$
\Delta \Phi=\frac{\Delta m^{2}}{2 E} L
$$

and

$$
L_{o s c}^{S}=2 \pi \frac{2 E}{\Delta m^{2}},
$$

where $\Delta m^{2}=m_{2}^{2}-m_{1}^{2}$, with $m_{2}>m_{1}$. To compute the oscillation probability, the following three assumptions are often applied [4]: $(i)$ The mass eigenstates are taken to be the energy (momentum) eigenstates, with a common energy (momentum); $(i i)$ up to $\mathcal{O}(m / E)$, we have the approximation $P \approx E>>m ;($ iii $)$ a massless trajectory is assumed, which means that the neutrino travels along the null trajectory defined by $d x=d t$. With these assumptions, the flavor state is simplified as

$$
\left|\nu_{\alpha}(x, t)\right\rangle=\sum_{j} U_{\alpha j} \exp \left[-i\left(\frac{m_{j}^{2}}{2 E}\right) x\right]\left|\nu_{j}\right\rangle .
$$

This state is then used to compute the oscillation amplitude.

\section{Reexamination of the Standard Treatments}

In this section, we discuss the standard procedures for the calculation of the phase factor along both the particle world-line and the null trajectory in Minkowski spacetime [30]. First, let us recall that, when the null condition is applied, the standard treatment yields

$$
\Phi^{S}=\int(E d t-P d x) \approx \int(E-P) d x=\frac{m^{2}}{2 E} L
$$

If the null condition $d x=d t$ is not used, we find

$$
\Phi=\int(E d t-P d x)=\int \frac{\left(E^{2}-P^{2}\right)}{P} d x=\frac{m^{2}}{P} L \approx 2 \Phi^{S} .
$$

The phase difference is then

$$
\Delta \Phi=\left(\frac{m_{2}^{2}}{P_{2}}-\frac{m_{1}^{2}}{P_{1}}\right) L \approx 2 \Delta \Phi^{S} .
$$

In order to illustrate the problem explicitly, the original definition of the phase factor will be obtained by means of the interval in the Minkowski spacetime. From Fig.1, we see 
that the interval of the world line, when the neutrino propagates from $\mathbf{A}$ to $\mathbf{B}$ in spacetime, is

$$
d s^{2}(A D)=\overline{B D}^{2}-\overline{B N}^{2}=(\overline{B D}+\overline{B N}) \overline{N D}=\frac{L^{2}}{v}-L^{2}=\frac{L^{2}}{v^{2} \gamma^{2}},
$$

and consequently

$$
\Phi=m d s(A D)=\frac{m L}{v \gamma}=\left(\frac{m^{2}}{E} L\right)\left(\frac{1}{v}\right) \approx 2 \Phi^{S} .
$$

The standard treatment gives

$$
m d s(A D)=\left(\frac{m \overline{B D}}{d s}\right) \overline{B D}-\left(\frac{m \overline{B N}}{d s}\right) \overline{B N}=(E-P) \overline{B N}=\frac{m^{2}}{2 E} L=\Phi^{S} .
$$

Although the difference $\overline{N D}=\overline{B D}-\overline{B N}$ between the particle world time and the null time is small, the phase is not solely related to the distance in space, but also to the interval in the spacetime, and the phase is sensitive to the null condition when the velocity of the neutrino approaches the speed of light. If we neglect this small difference in the time interval, a factor of 2 will appear in the phase factor! To illustrate this conclusion, we inspect in the next subsections the standard treatments in more detail.

\subsection{Reexamination of the Same-Energy Prescription}

If two neutrinos share the same energy $\left(E_{1}=E_{2}=E, E^{2}-P_{j}^{2}=m_{j}^{2}\right)$, but present different momenta, the contribution to the phase difference will come from the integration of the momentum in space because the same-energy condition makes the integration of the energy in time to vanish. This is the main point of the same-energy condition in the standard treatment. Now, we will explore this point in more detail by using the Minkowski diagram of Fig.2.

For convenience, we will keep using the convention $m_{2}>m_{1}$, which leads to $\gamma_{1}>\gamma_{2}$, $P_{1}>P_{2}$ and $v_{1}>v_{2}$. In the Minkowski diagram of Fig.2, the faster the frame the closer it is of the null line [30]. This property will be helpful for our analysis.

According to the standard treatment in the case of same-energy $\left(E_{1}=E_{2}\right)$, we have

$$
\begin{aligned}
\Delta \Phi^{S} & =\int_{A}^{B}\left(E_{2} d t-P_{2} d x\right)-\int_{A}^{B}\left(E_{1} d t-P_{1} d x\right) \\
& =-\int_{A}^{B}\left(P_{2}-P_{1}\right) d x=\frac{\Delta m^{2}}{2 E} L
\end{aligned}
$$

This computation seems not to use the null condition $d x=d t$, and it does not use the fact that, because the velocities are not the same, the neutrinos with velocities $v_{1}$ and $v_{2}$ follow different world-lines. However, the null condition is in fact used when we replace both $\overline{B D_{1}}$ and $\overline{B D_{2}}$ by $\overline{B N}$ (see Fig.2). It is thus important to remark that it is the null condition, not the same energy condition, that accounts for the cancellation of the time phase. 
What does it happen if the real world-lines (geodesic) of the neutrinos are taken into account? In order to answer this question, it is important to notice that the massive neutrinos $\nu_{1}$ and $\nu_{2}$ describe two different world lines from the source $\mathbf{A}$ to the detector $\mathbf{B}$, given respectively by $A D_{1}$ and $A D_{2}$ (see Fig.2). Taking this into account, we obtain:

$$
\begin{aligned}
\Delta \Phi & =\int_{A}^{D_{2}}\left(E_{2} d t-P_{2} d x\right)-\int_{A}^{D_{1}}\left(E_{1} d t-P_{1} d x\right) \\
& =\int_{D_{1}}^{D_{2}} E d t-\int_{A}^{B}\left(P_{2}-P_{1}\right) d x \\
& =\frac{\Delta m^{2} E}{2 P_{1} P_{2}} L+\frac{\Delta m^{2}}{2 E} L \\
& \approx \frac{\Delta m^{2}}{2 E} L+\frac{\Delta m^{2}}{2 E} L=2 \Delta \Phi^{S} .
\end{aligned}
$$

If the arrival time-difference of the neutrinos is considered, therefore, the time-phase is not canceled. As it is as large as the space-phase, the real phase results twice the value yielded by the standard treatment. A similar conclusion has also been obtained in Ref. [9, 28]. The computation of (18) and (19) indicates that it is the null condition, not the same energy condition, the responsible for the duplication of the standard phase difference.

\subsection{Reexamination of the Same-Momentum Prescription}

Following a procedure similar to that used in the case of the same-energy prescription, we examine now the same-momentum prescription $\left(P_{1}=P_{2}=P, E_{j}^{2}-P^{2}=m_{j}^{2}\right)$. We still suppose $m_{2}>m_{1}$, which leads to $\gamma_{1}>\gamma_{2}, E_{1}<E_{2}$ and $v_{1}>v_{2}$. These relations allow us to use the same Minkowski diagram of Fig.2. We find in this case

$$
\begin{aligned}
\Delta \Phi & =\int_{A}^{D_{2}}\left(E_{2} d t-P_{2} d x\right)-\int_{A}^{D_{1}}\left(E_{1} d t-P_{1} d x\right) \\
& =\int_{B}^{D_{2}} E_{2} d t-\int_{B}^{D_{1}} E_{1} d t \\
& =\frac{\Delta m^{2}}{P} L \approx 2 \Phi^{S} .
\end{aligned}
$$

It is the same result of the case of the same-energy prescription. However, a small difference exists. In order to show the influence of the same-momentum and of the nullcondition on the calculation of the phase difference, we examine the phase computation of the same-momentum prescription of the standard treatment, but using the null condition. This amounts to replace $B D_{2}$ and $B D_{1}$ by the null time corresponding to $B N$. We find

$$
\begin{aligned}
\Delta \Phi^{S} & =\int_{A}^{B}\left(E_{2} d t-P_{2} d x\right)-\int_{A}^{B}\left(E_{1} d t-P_{1} d x\right) \\
& =\int_{B}^{N} E_{2} d t-\int_{B}^{N} E_{1} d t \\
& =\int_{B}^{N}\left(E_{2}-E_{1}\right) d x=\frac{\Delta m^{2}}{2 P} L=\Phi^{S}
\end{aligned}
$$


We see in this way that the same-momentum condition cancels the space phase in both (20) and (21). However, the time-phase in (20) is twice the value found in (21).

A problem then arises: Why do both the same-energy and the same-momentum prescriptions yield the same practical result? A possible answer is that the time-phase and the space-phase might be equivalent when the null condition is used, as already concluded in Ref. [9]. Somehow, the null condition implies in neglecting the arrival time-difference of the two neutrinos. If this is correct, we should get $\Phi^{S}$ when computing the phase factor under the assumption of the same-velocity prescription, as in this case no arrival time-difference exists.

\subsection{On the Same-Velocity Prescription}

Although the same velocity descrition $[24,25,26]$ is forbidden by the kinematical consideration [27], here, we still explore in the Minkowski diagram how it arises the standard oscillation phase.

Instead of supposing $E_{1}=E_{2}$ or $P_{1}=P_{2}$, let us suppose the same-velocity prescription for the neutrinos motion $[24,25,26]: v_{1}=v_{2}\left(\gamma_{1}=\gamma_{2}\right)$. We have in this case

$$
\frac{m_{2}}{m_{1}}=\frac{E_{2}}{E_{1}}=\frac{P_{2}}{P_{1}}
$$

and

$$
\frac{P_{1}}{E_{1}}=\frac{P_{2}}{E_{2}} .
$$

The phase difference can be computed along the world line shown in Fig.1. The result is

$$
\begin{aligned}
\Delta \Phi & =\int_{A}^{D}\left(E_{2} d t-P_{2} d x\right)-\int_{A}^{D}\left(E_{1} d t-P_{1} d x\right) \\
& =\int_{B}^{D} \frac{\left(P_{2}-P_{1}\right)}{v_{o}}\left(\frac{d x}{v_{o}}\right)-\int_{A}^{B}\left(P_{2}-P_{1}\right) d x \\
& =\frac{\left(P_{2}-P_{1}\right)}{\gamma^{2} v_{o}^{2}} L=\frac{\left(P_{2}^{2}-P_{1}^{2}\right)}{\gamma^{2} v_{o}^{2}\left(P_{2}+P_{1}\right)} L \\
& =\frac{\Delta m^{2}}{\left(P_{2}+P_{1}\right)} L \approx \Delta \Phi^{S} .
\end{aligned}
$$

Like the same-energy and the same-momentum prescriptions, the same-velocity prescription gives exactly the value of the standard treatment. We conclude in this way that the confusion on the understanding of the neutrino phase factor has its origin in the arrival-time difference. The null condition of the standard treatment includes the information of the same speed of the two neutrinos. 


\section{4 geodesic phase and null phase in the curved space- time}

As discussed in the flat spacetime, calculating the phase along the geodesic will produce a factor of 2, now we can also obtain this conclusion in the general curved spacetime, not only in the Schwarzschild spacetime [10]. The velocity of an extremely relativistic neutrino is nearly the speed of light in the curved spacetime. Although seemingly irrelevant to think about the difference between the geodesic and the null, this tiny deviation becomes important for the understanding of the neutrino oscillation. Motivated by this argument, we will compare the neutrino phase when calculated along the geodesic and along the null-line. With this, we will be able to verify the factor of 2 when the null is replaced by the geodesic. This study can be shown to remain valid in the case of the flat spacetime.

Let $n^{\mu}$ and $\stackrel{\circ}{n}^{\mu}$ be the tangent vectors to the geodesic and to the null-line, respectively, their difference $\epsilon^{\mu}$ being a small quantity for the case of an extremely relativistic neutrino. Here, we suppose that the two neutrinos, the massless and massive, start their journey at the same initial spacetime position A, and their space routes are almost the same. But, there will be an arrival time-difference at the detector position B. This means that their 4-dimensional spacetime trajectories are not the same, and consequently the tangent vectors will present a small difference. Thus, we have

$$
n^{\nu}=\stackrel{\circ}{n}^{\nu}+\epsilon^{\nu}, \quad \text { or } \quad P^{\nu}=\stackrel{\circ}{P}^{\nu}+m \epsilon^{\nu},
$$

where $P^{\nu}=m n^{\nu}\left(\stackrel{\circ}{P}^{\nu}=m n^{\nu}\right)$ is the 4-momentum along the geodesic (null-line) with

$$
\stackrel{\circ}{n}^{\nu}=\frac{d x^{\mu}}{d \lambda}=\frac{d \stackrel{\circ}{x}^{\nu}}{d s}
$$

and

$$
n^{\mu}=\frac{d x^{\mu}}{d s} .
$$

In these expressions, $\lambda$ and $s$ are respectively affine parameters along the null and the geodesic lines. These two tangent vectors satisfy the mass shell relations of the geodesic and the null-line:

$$
g_{\mu \nu} n^{\mu} n^{\nu}=1
$$

and

$$
g_{\mu \nu} \stackrel{\circ}{n} \stackrel{\circ}{n}^{\nu}=0 .
$$

Now, substituting (25) into (28), we obtain

$$
g_{\mu \nu}\left(\stackrel{\circ}{n}^{\mu}+\epsilon^{\mu}\right)\left(\stackrel{\circ}{n}^{\nu}+\epsilon^{\nu}\right)=1,
$$


or, by using (29),

$$
2 g_{\mu \nu} \stackrel{\circ}{n}^{\mu} \epsilon^{\nu}+\mathcal{O}\left(\epsilon^{2}\right)=1
$$

We can estimate the order of $\left\{n^{\mu}\right\}$ and $\left\{\stackrel{\circ}{n}^{\mu}\right\}$ by noting that $n \epsilon \sim 1 / 2$, which implies that $\epsilon \sim n^{-1} \sim \frac{m}{E}$, where $E=P^{o} \sim P^{i}(i=1,2,3)$ for a relativistic neutrino.

The neutrino phase induced by the null condition, as in the standard treatment, comes from the 4-momentum $P^{\nu}$ defined along the geodesic line, and the tangent vector $\left\{\stackrel{\circ}{n}^{\mu}\right\}$ to the null-line [6]. We notice that, if the 4-momentum $\stackrel{\circ}{P}^{\nu}$ defined along the null-line was instead used to compute the null phase, we would obtain zero because of the null condition. Therefore, the phase along the geodesic line (geodesic phase) and the phase along the null-line (null phase) can be written respectively as $[6,31,32,33]$

$$
\Phi(\text { geod })=\int m d s=\int g_{\mu \nu} P^{\mu} n^{\nu} d s
$$

and

$$
\Phi(\text { null })=\int g_{\mu \nu} P^{\mu} n^{\nu} d s .
$$

Therefore, the difference between the geodesic phase and the null phase, by using Eq.(31), is

$$
\begin{aligned}
\Phi(\text { geod }) & -\Phi(\text { null })=\int g_{\mu \nu} P^{\mu}\left(n^{\nu}-\stackrel{\circ}{n}^{\nu}\right) d s \\
& =\int g_{\mu \nu} P^{\mu} \epsilon^{\nu} d s=\int g_{\mu \nu} \stackrel{\circ}{ }^{\mu} \epsilon^{\nu} d s+\mathcal{O}\left(\epsilon^{2}\right) \\
& =\frac{1}{2} \int m d s+\mathcal{O}\left(\epsilon^{2}\right)=\frac{1}{2} \Phi(\text { geod })+\mathcal{O}\left(\epsilon^{2}\right)
\end{aligned}
$$

that is

$$
\Phi(\text { geod })=2 \Phi(\text { null })+\mathcal{O}\left(\epsilon^{2}\right)
$$

This conclusion, valid for a general curved spacetime, is similar to that found in in a Schwarzschild [10] spacetime. Concerning the Schwarzschild spacetime, Bhattacharya et al [10] have the following argument for the factor of 2. As the neutrino energy is fixed, but the masses are different, if an interference is to be observed at the same final spacetime point $\mathrm{B}\left(r_{B}, t_{B}\right)$, the relevant components of the wave function could not both have started at the same initial spacetime point $\mathrm{A}\left(r_{A}, t_{A}\right)$ in the semiclassical approximation. Instead, the lighter mass (hence faster moving) component must either have started at the same time from a spatial location $r<r_{A}$, or (what is equivalent) started from the same location $r_{A}$ at a later time $t_{A}+\Delta t$. Hence, there is already an initial phase difference between the two mass components due to this time gap, even before the transport from $r_{A}$ to $r_{B}$ which leads to the phase $\Phi$ (null), i.e., the additional initial phase difference may be taken into account [10]. 


\section{5 discussions and conclusions}

As mentioned above, the same-energy and the same-momentum prescriptions in calculating the mass neutrino interference phase will obtain the same result if the arrival time difference is taken into account, which will be, mathematically, equivalent to the phase of a treatment by using the same-velocity condition. However, we remark that generally neither energy nor momentum are equal in the factual physical process $[17,18]$, the standard treatment of the same-energy and the same-momentum prescriptions of massive neutrino phases is just a mathematical simplification in some sense when applying the plane wave approximation. Then our analysis of the massive neutrino standard phases in the Minkowski diagram indicates that the same phase factor will be obtained if the two massive neutrinos follow the null line or possess the same velocity. If the phase calculated along the particle world line, the realtive phase will produce a factor of 2 [28], the reason is that we despise the arrival time difference of the two mass neutrinos, which results in a double counting effect. This conclusion is correct in both flat spacetime and the curved spacetime. Further, for the better understanding of the mass neutrino interference, we should consider the neutrino mixing state as a wave-packet $[17,29]$, or a relativistic quantum ball. According to this scheme, two physical aspects must be considered. On one hand, there is the classical trajectory of the ball which is defined by giving the initial conditions in the classical sense. This motion is relativistic, leading thus to the relativity of the simultaneity as the velocities of the different mass neutrinos are not the same. The classical trajectory, therefore, is defined in terms of macroscopic quantities of the packet, the quantum average of quantities, in accordance with the correspondence principle of quantum mechanics. In the classical sense, the average mass and velocity of the packet ball define its orbit. The distance from the source to the detector is usually measured accurately, which is a classical measurement with a macroscopic precision. On the other hand, there is the quantum dynamics of the packet, which is a two-state system like a neutral kaon or a B meson. This fuzzy dynamics takes place in the microscopic scale $d$, the characteristic dimension of the ball, which is small compared to the dimension $L$ of the macroscopic trajectory. This quantum dynamics, that is, the internal evolution of the packet, is well described and understood in the particle physics context.

As a final comment, we would like to argue that, if the individual mass-eigenstate is supposed to have a well defined classical velocity, its orbit would be well defined at any spacetime position. This, however, is not in accordance with the quantum interference description, which consequently would never occur. This means that the quantum fuzzy is necessary for the wave-packet, a well defined orbit being valid only for the classical (macroscopic) quantum average. According to this point of view, two basic points concerns the description of the neutrino oscillation. For the kinematics, we use the plane-wave description with a Lorentz invariant phase. For the dynamics, we use the wave-packet and fuzzy 
quantum path. We use, therefore, two concepts for the neutrinos, particle and wave, classical and quantum. The neutrino is described in terms of a spinor wave-packet, and the spacetime translation induces the spinor to precess in the state vector-space. In other words, we take the mixing state as a classical object propagating along the classical world-line with a well defined velocity - the group velocity. The individual mass-eigenstates propagate with a phase velocity, and only the relative phase velocity will produce the realistic interference phenomenon.

\section{Acknowledgments}

Thanks are due to the scientific visiting support by ICTP, Trieste, Italy. A.B. is supported by NRF of South Africa. Discussions with A. Smirnov are highly appreciated. The authors are grateful to the modification suggestions from the anonymous referee.

\section{References}

[1] K. Zuber, Phys. Rep. 305, 295 (1998).

[2] S. Bilenky, C. Giunti and W. Grimus, Prog. Part. Nucl. Phys. 43, 1 (1999). (hep$\mathrm{ph} / 9812360)$.

[3] S. Pakvasa, Neutrinos (hep-ph/9804426).

[4] S. Bilenky and B. Pontecorvo, Phys. Rep. 41 (1978) 225; Rev. Mod. Phys. 59, 671 (1987).

[5] F. Boehm and P. Vogel, Physics of Massive Neutrinos (Cambridge University Press, Cambridge, 1992).

[6] C.Y. Cardall and G.M. Fuller, Phys. Rev. D55, 7960(1996).

[7] N. Fornengo, C. Giunti, C. W. Kim, and J.Song, Phys. Rev. D56, 1895 (1997).

[8] Y. Kojima, Mod. Phys. Lett. A 11, 2965 (1996).

[9] Y. Grossman and H. J. Lipkin, Phys. Rev. D. 55, 2760 (1997).

[10] T. Bhattacharya, S. Habib, and E. Mottola, Phys. Rev. D59, 067301 (1999).

[11] Pereira, J.G., and Zhang, C.M., Gen. Rel. Grav., 32, No. 8. 1633 (2000); 33, No. 12, 2801 (2001). 
[12] Zhang, C.M., Nuovo Cimento B, 115, No.4, 437-444 (2000).

[13] C.M. Zhang, and A. Beesham, Gen. Rel. Grav., 33, No. 6., 1011 (2001).

[14] A. Dolgov, A. Morozov, L. Okun, and M. Schepkin, Nucl. Phys. B502, 3 (1997).

[15] L.B. Okun, Surveys in High Energy Physics, 15, 75 (2000).

[16] I.S. Tsukerman, JETP Lett. 73, 380 (2001); Pisma Zh.Eksp.Teor. Fiz. 73, 424 (2001).

[17] A.D. Dolgov, hep-ph/0004032, A lecture presented at 7th Course: Current Topics of Astrofundamental Physics, Erice-Sicile, 5-16 Decemebr, 1999.

[18] C. Giunti, Mod. Phys. Lett. A16, 2363 (2001), hep-ph/0104148.

[19] B. Kayser, Phys. Rev. D24, 110 (1981).

[20] R.G. Winter, Lett. Nuovo Cimento, 30, 101 (1982).

[21] T. Goldman, Source dependence of neutrino oscillations (hep-ph/9604357).

[22] H. Lipkin, Phys. Lett. B 348, 604 (1995).

[23] M.M. Nieto, Quantum interference: from kaons to neutrinos (hep-ph/9509370).

[24] Y. Takeuchi, Y. Tazaki, S.Y. Tsai and T. Yamazaki, Mod. Phys. Lett. A14, 2329, (1999).

[25] S. De Leo, G. Ducati, and P. Rotelli, hep-ph/9906460.

[26] Y. Takeuchi, Y. Tazaki, S.Y. Tsai, and T. Yamazaki, hep-ph/0006334.

[27] L.B. Okun, I.S. Tsukerman, Mod. Phys. Lett. A15, 1481 (2000), hep-ph/0007262

[28] H. Lipkin, "Quantum Mechanics of Neutrino Oscillations - Hand Waving for Pedestrians", (hep-ph/9901399).

[29] C. Giunti, C.W. Kim and U.W. Lee, Phys. Rev. D44, 3635 (1991).

[30] A.P. French, Special Relativity (Norton \& Company, New York, 1969).

[31] L. Stodolsky, Gen. Rel. and Grav., 11, 391 (1979).

[32] J. Audretsch, J. Phys. A, 14, 411 (1981).

[33] J. Anandan, Phys. Rev. D 15, 1448 (1977);

J. Anandan, IL NUOVO CIMENTO 53A, 221 (1979). 
Figure 1: For the case of same-velocity neutrinos, the Lorentz transformation makes the moving axis inclined to the null-line. The primed coordinate frame represents the neutrino system moving with velocity $v$ in relation to the laboratory system $(x, t)$.

Figure 2: For the case of different-velocity neutrinos, different Lorentz transformations make the corresponding coordinate system differently inclined to the null-line. The faster the object the closer they are of the null-line. The primed and double primed coordinate frames represent respectively neutrinos $\nu_{1}$ and $\nu_{2}$ with velocities $v_{1}$ and $v_{2}$ relative to the laboratory system $(x, t)$. 


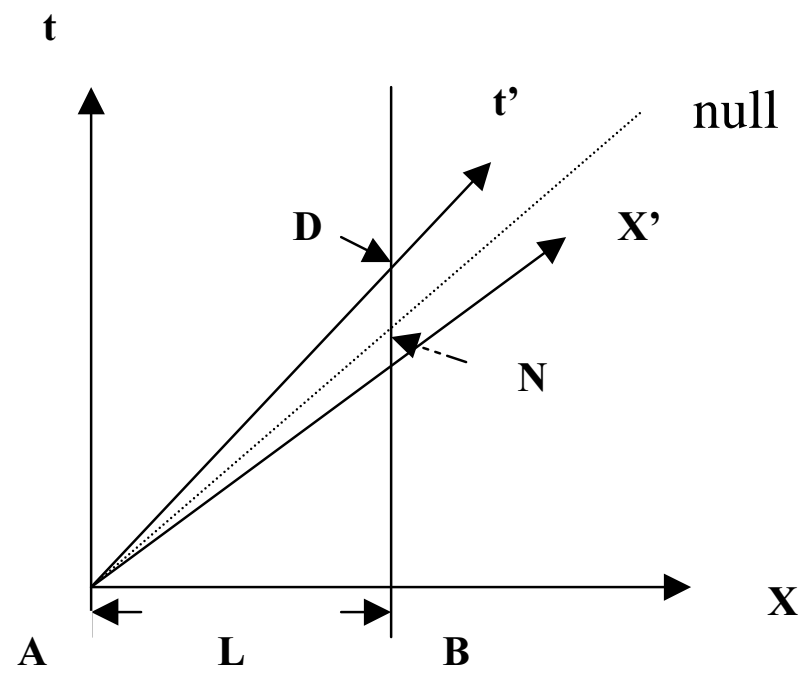





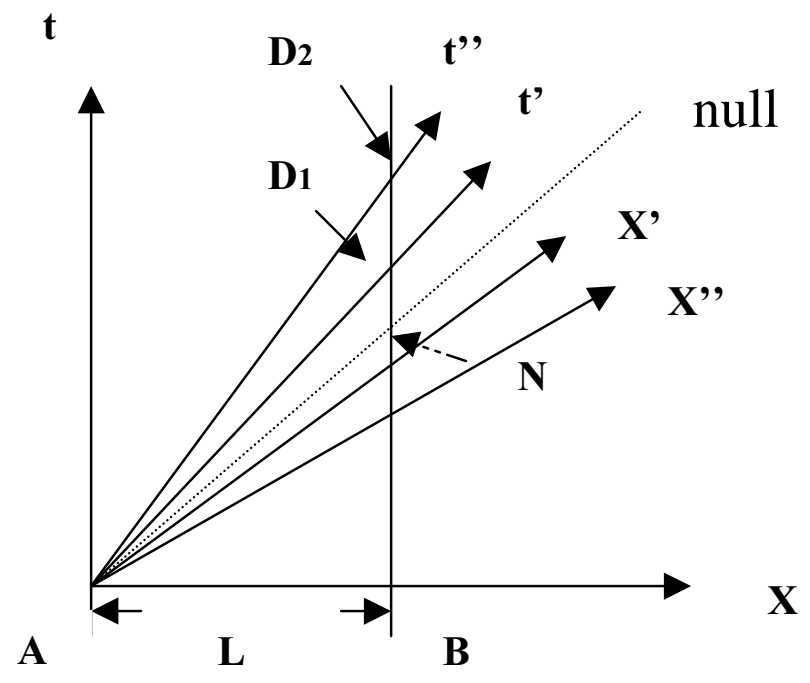


